\title{
Nontraumatic bilateral atypical femoral fractures associated with long-term alendronate therapy
}

\author{
Olayinka A. Ogundipe*
}

Department of Medicine of the Elderly, Royal Infirmary of Edinburgh, Little France Crescent, Edinburgh, Scotland

Received: 02 September 2020

Accepted: 05 October 2020

*Correspondence:

Dr. Olayinka A. Ogundipe,

Email: ola_ayodele@hotmail.com

Copyright: () the author(s), publisher and licensee Medip Academy. This is an open-access article distributed under the terms of the Creative Commons Attribution Non-Commercial License, which permits unrestricted non-commercial use, distribution, and reproduction in any medium, provided the original work is properly cited.

\begin{abstract}
Alendronate is a bisphosphonate commonly used in the treatment of post-menopausal and steroid-associated osteoporosis. Bisphosphonates have an evidence base for reducing the occurrence of typical osteoporotic fractures. However, there has been growing recognition of a correlation with the use of long-term therapy with bisphosphonates, and rare occurrence of atypical femoral fractures (AFFs). This report describes a 72-year-old caucasian woman presenting with evolving groin and thigh pains of two weeks duration. Plain X-rays noted features compatible with bilateral impending femoral subtrochanteric fractures. She had been taking oral alendronate $70 \mathrm{mg}$ weekly for ten consecutive years as treatment for osteoporosis. Based on the medication history, the absence of preceding trauma or a fall, and the presence of supportive radiological findings, the diagnosis was made of impending bilateral proximal femur fractures secondary to long-term bisphosphonate therapy. The alendronate was discontinued, and the patient managed with two planned successive surgeries involving the insertion of intertrochanteric antegrade nails (interTAN) to both femurs. Following a period of rehabilitation, she was successfully discharged home. Some pharmacokinetic and pharmacodynamic considerations of bisphosphonates are discussed. The write-up presents a brief literature review of AFFs. The index report is further reviewed in relation to the American society for bone and mineral research (ASBMR) task force's recommended case definition of what constitutes AFFs. The discussion concludes with the application of two previously validated causality assessment systems (CAS). In this instance, both CAS indicated a 'probable' classification for the adverse drug reaction (ADR) to prolonged usage of oral alendronate.
\end{abstract}

Keywords: Adverse drug reaction, Alendronate, Atypical femoral fractures, Bisphosphonates, Causality, Pharmacovigilance

\section{INTRODUCTION}

Bisphosphonates are a class of drugs with pharmacological properties used in the prevention and treatment of osteoporosis. ${ }^{1,2}$ Bisphosphonates are also used in the management of some other disorders of bone, and/or abnormalities of bone profile related biochemistry (e.g. Paget's disease, primary hyperparathyroidism, hypercalcaemia associated with malignancies, etc). ${ }^{1}$ Furthermore, bisphosphonates have a supportive evidence base for the reduction of osteoporotic fractures in both vertebral and nonvertebral sites. ${ }^{1-3}$

Alendronic acid is one of the more commonly prescribed oral bisphosphonates. ${ }^{1-3}$ The bisphosphonates have been recognised to have risks of uncommon to rare adverse effects. ${ }^{1}$ One such association is the occurrence of atypical femoral fractures (AFFs) which are rare occurrences linked to the long-term use of bisphosphonates. ${ }^{1,4}$ These fractures are located along the femoral shaft, occur in association with minimal or no trauma, and also have characteristic radiological features 
and clinical symptomatology which are not typical of fractures of osteoporotic nature. ${ }^{4}$

This case report describes an older female patient who presented with clinical symptoms and plain radiological features consistent with bilateral AFFs. These were noted to have occurred in the context of a preceding prolonged (ten year) use of oral alendronic acid.

\section{CASE REPORT}

A 72-year-old caucasian woman was referred to hospital with a two-week history of having experienced a feeling of her legs giving way while walking one day, and subsequent pain in her left groin. There was no reported fall nor trauma. The left groin pain radiated down her thigh and towards her left knee. She was still able to mobilise but was finding this to be increasingly difficult due to the pain.

In the days leading up to the admission, she started to experience new onset of discomfort and pain in the proximal right thigh, most notable when she attempted weight-bearing. She felt this was due to having to redistribute her weight from the more painful left lower limb, onto the right side.

On admission, she was noted to have normal observations and appeared well. There was no clinical suggestion of significant lower limb length discrepancy, nor signs of malrotation. Her weight was $62 \mathrm{~kg}$, height $147 \mathrm{~cm}$, and her derived body mass index (BMI) was $28.69 \mathrm{~kg} / \mathrm{m}^{2}$.

Her medication history noted oral omeprazole $20 \mathrm{mg}$ gastroresistant capsules taken once daily, as well as combined supplements of oral calcium (1 gm/day) and vitamin D (800 units/day). In addition, she had been taking oral alendronate $70 \mathrm{mg}$ once weekly for the preceding consecutive ten years. The indication for the bisphosphonate was bone protection on account of prior long-term steroid (prednisolone) therapy for polymyalgia rheumatica (PMR). Lumbar spine osteoporosis that had been confirmed on a dual energy X-ray absorptiometry (DEXA) scan undertaken 3 years prior to this admission.

She was a non-smoker and drank minimal alcohol. Other than PMR, her medical history was unremarkable and there was no history of malignancy.

Her admission blood profile was normal for full blood count, urea and electrolytes, estimated glomerular filtration rate (eGFR), serum calcium, and serum phosphate. Her serum $25-\mathrm{OH}$ vitamin D level was replete at $65 \mathrm{nmol} / \mathrm{L}$ (reference $25-162$ ).

Plain X-rays on admission noted features consistent with bilateral impending/imminent femoral fractures (Figure 1).
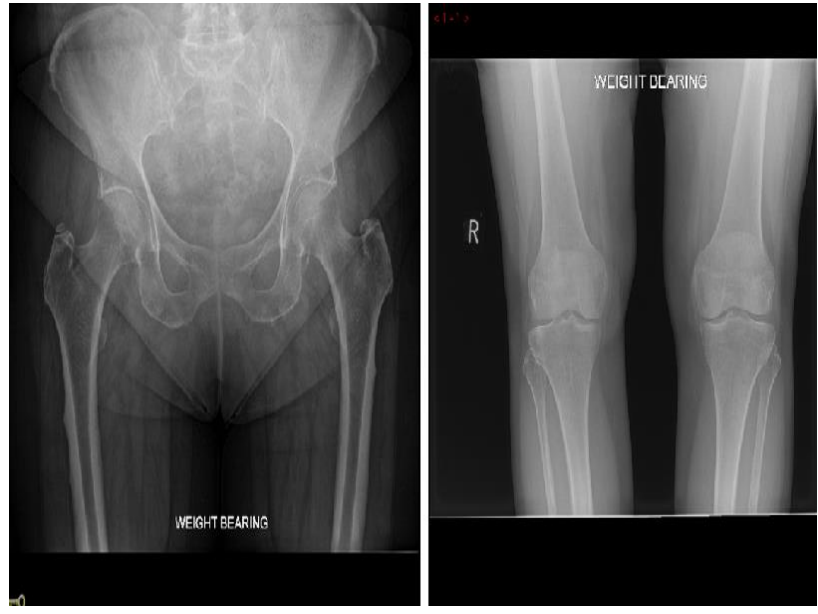

Figure 1: Plain $X$-ray views of the $(\mathrm{A})$ proximal femurs, showing signs of 'beaking' to the lateral cortices; evidence of subtrochanteric lesions and impending femoral fractures. (B) The distal femurs, knees and proximal tibia + fibular bones; normal appearances.

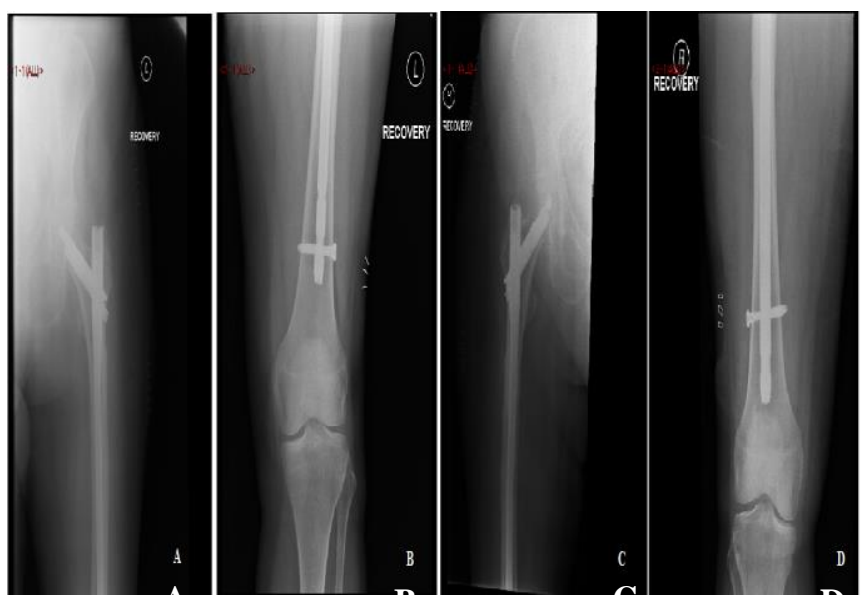

Figure 2: Plain X-ray views of the (A) left proximal femur (B) left distal femur (C) right proximal femur

(D) right distal femur, immediate post-operative image following insertion of inter-TAN nails.

In the absence of a fall or trauma, plus the relevant context of prolonged therapy with a bisphosphonate (alendronate), and given the supportive plain X-ray findings, the diagnosis was made of impending bilateral proximal femur pathological fractures.

The bisphosphonate therapy was discontinued. Following specialist orthopaedic surgical assessments, she had planned sequential orthopaedic surgeries which were scheduled one week apart. Both operations were completed under spinal anaesthesia with nerve blocks, and involved the insertion of prophylactic intertrochanteric antegrade nail (inter-TAN) to both femurs (Figure 2). There were no post-operative complications. She made good progress over a short 
period of inpatient rehabilitation and was subsequently discharged home.

\section{DISCUSSION}

Bisphosphonates are pharmacological agents described as possessing inhibitory activity against the function of osteoclasts, and thereby reducing bone resorption and bone turnover. ${ }^{1-3}$ Over the short to intermediate term, the cumulative effect of bisphosphonate use is to increase bone mass by preventing bone remodelling..$^{4-7}$ As a result, the bone mineral density (BMD) may increase due to inhibition of bone tissue breakdown (osteoclastic function). ${ }^{1,3,5-7}$ However, innate to this process are qualitative changes to the bone tissue, which present at least a theoretical potential for increased fracture risk over the long term. ${ }^{5-7}$ The possibility of atypical fracture occurrence in the long term may arise if overall bone integrity is compromised by impaired remodelling of damaged or effete microscopic bone tissue by the inhibited action of osteoclasts. ${ }^{4,6,7}$

Alendronate sodium (or alendronic acid) is one of the frequently prescribed and first-line oral options used in the treatment of post-menopausal osteoporosis. ${ }^{1}$ Alendronic acid is also employed in the treatment of male osteoporosis. ${ }^{1}$ It is also used in the prevention and treatment of steroid associated osteoporosis. ${ }^{1-3}$ In addition, .bisphosphonates also have a documented evidence-base for the reduction of osteoporotic fractures. ${ }^{2-}$

${ }^{4}$ Aside from osteoporosis, bisphosphonates have potential complementary roles in the management of other clinical conditions such as Paget's disease, primary hyperparathyroidism, and significant hypercalcaemia linked to metastatic bone disease. ${ }^{1}$

Some basic pharmacodynamic and pharmacokinetic considerations of alendronic acid bisphosphonates are available as oral and parenteral (intravenous/IV) formulations. ${ }^{1}$ Alendronic acid exists in an oral formulation, and is available as either a $10 \mathrm{mg}$ once daily dose, or as a $70 \mathrm{mg}$ once weekly dose. ${ }^{1}$ The oral medicinal forms of alendronic acid are available as tablets, effervescent tablets, and as an oral solution. ${ }^{1}$

Guidance on taking alendronic acid recommends that the (standard) tablets should be swallowed whole, whereas the oral solution should be swallowed as a single measured dose. ${ }^{1}$ Alendronic acid is also advised to be taken with a large volume of plain water (mineral water is not recommended) while in a sitting or standing position. ${ }^{1}$ The medication should be taken on an empty stomach at least 30 minutes prior to the patient's breakfast (or at least 30 minutes before .taking other medications). ${ }^{1}$ Once swallowed, patients are advised to sit upright or stand for at least 30 minutes following its consumption. ${ }^{1}$

Alendronate contains a nitrogen-containing biochemical structure. ${ }^{6}$ It has very poor oral bioavailability with some estimates citing this to be in the order of $<1 \%$ (hence the advice to swallow on an empty stomach). ${ }^{1}$ Once absorbed, bisphosphonates have a very long duration of action, with estimates of the half-life of alendronate deemed to in the order of $>5$ years, i.e. once effective skeletal binding has been achieved. ${ }^{6}$ The drug is minimally metabolised, and is renally excreted in a largely unchanged state. ${ }^{1,6}$ The general recommendation is to avoid alendronic acid in patients with chronic kidney disease, if their eGFR is less than $35 \mathrm{ml} /$ minute $/ 1.73 \mathrm{~m}^{2}$.

\section{Diagnosing AFFs}

Although bisphosphonates are regarded as being effective treatment options for osteoporosis and other conditions as described above, there is a growing recognition of the rare occurrence of AFFs with prolonged bisphosphonate treatment. $^{8-11}$ These AFFs occur mainly (but not exclusively) in patients receiving long-term treatment for osteoporosis. ${ }^{5,7,8-12}$ As a result of this, patients should routinely be advised to seek medical advice and/or to report the occurrence of any new or evolving symptoms such as groin, hip or thigh pains while they are taking bisphosphonates.

Aside from AFFs, other reported uncommon to rare (relative to overall usage) bone related adverse drug reactions (ADRs) or complications of bisphosphonate use are osteonecrosis of the jaw (dental osteonecrosis), and defects or deficiencies of the dental enamel. ${ }^{1,13}$ Osteonecrosis of the external auditory canal has also been reported as another rare occurence. ${ }^{1}$ The risks of dental osteonecrosis appear to be greater in patients receiving IV forms of bisphosphonates (e.g. as part of the treatment of cancer), compared to the noted lower occurrences in patients receiving oral bisphosphonate therapies (e.g. for osteoporosis or Paget's disease). ${ }^{1}$ This might be partly indicative of the greater bioavailability linked to the administration of IV compared to oral formulations of bisphosphonates. Some previously described risk factors for developing osteonecrosis of the jaw include: the potency of the bisphosphonate used (e.g. possibly highest for IV zoledronate), the route of administration (IV versus oral), the cumulative dose received, the type and duration of the malignancy, other concomitant treatment(s), smoking status, presence of other comorbid conditions, and positive history of dental disease. ${ }^{1}$ The importance of dental status reviews and dental check-ups has been highlighted. Where relevant (e.g. existing dental disease), dental input may be required before instituting bisphosphonate therapy, or alternatively a dental review may be required as soon as possible thereafter (e.g. if new clinical concerns are noted or reported). ${ }^{1,13}$

In addition, the continuing prescription and use of regular bisphosphonate therapy (e.g. for osteoporosis) should be subject to review guided by a periodic re-assessment of the benefits and risks to individualised patients. The need to review the pros and cons of continuing bisphosphonate therapy appears to be especially relevant to those taking these medicines for more than five consecutive years. The 
rationale for this is the mounting body of evidence indicating that the incidence of AFFs appear to be greater with prolonged durations ( $>5$ to 10 years) of treatment. $5,10,12$

Some reports have identified that patients of Asian descent may be more susceptible to AFFs than persons of Caucasian descent. ${ }^{4,5}$ There is also some evidence that the relative risk of AFFs reduces following discontinuation of regular bisphosphonate use, even in those who have taken the medication for prolonged periods. Any patient suspected to have AFFs should be considered for discontinuation of bisphosphonate treatment. This should take place alongside the re-evaluation of their bone health to inform a clinical decision as to whether or not they may benefit from a bisphosphonate 'drug holiday,' or a switch to alternative treatment options if deemed clinically appropriate to the individual patient. ${ }^{1,2,4}$

In general, AFFs are dissimilar in nature from more typical stress fractures as they have a tendency to begin on the lateral femoral cortex. ${ }^{6,14-16}$ AFFs tend to occur in areas that correspond to focal points of high tensile stress during activities like walking. In distinction, typical stress fractures have a tendency to begin on the medial aspect of the femoral cortex. The latter in turn corresponding to areas or focal points that bear greatest compression strain during physical activities like running. 5,6,8,16

In 2010, in an attempt to standardise the case ascertainment of AFFs, the American society for bone and mineral research (ASBMR) task force described several criteria for use in case definition. ${ }^{14}$ Over time, the criteria and the case definition of AFFs have undergone iterative reviews and was updated by the Task Force in 2013, producing a report published in 2014. ${ }^{15}$ In addition, review of the wider medical literature notes that the specified radiological criteria have also been further evaluated by other researchers. ${ }^{16}$

For example, applying the updated ASBMR Task Force's second report definition of AFFs, the initial inclusion criterion (effectively a 'required feature') is that the fracture must be a femoral shaft fracture (FSF). ${ }^{15}$ By this definition, the FSF must occur along the diaphysis of the femur, and is generally situated distal to the lesser trochanter (subtrochantric region) and proximal to the supracondylar 'flare' (or supracondylar region). ${ }^{15}$

If the suspected case meets the 'required feature' clause, then the case definition of AFFs further requires that a minimum of 4 out of 5 pre-defined 'major' features must also be present. ${ }^{15}$

\section{The major features or criteria include}

1) Trauma - an association with minimal or no trauma (e.g., falling from a standing height or less; or no trauma / no fall) 2) configuration of the fracture - The fracture line originates at the lateral cortex (corresponding to the areas that sustain the most tensile stress during standing or walking). If untreated, the fracture then progresses medially towards becoming a complete fracture - and often appearing as 'a substantially transverse orientation.' However, the updated case definition gives allowance for the fact that the fracture line might appear to be 'oblique' (rather than 'transverse') i.e. as the fracture line progresses medially 3) completeness of the fracture complete fractures traverse both femoral cortices and may have a medial spike, whereas incomplete fractures involve the lateral femoral cortex only 4) comminution - The fracture is either minimally comminuted or noncomminuted in appearance 5) periosteal or endosteal thickness - localised endosteal or periosteal thickening of the lateral cortex is present at the site of the fracture (an appearance also called or described as "beaking" or "flaring")

In addition, the following minor features or criteria may sometimes be present or noted, but are not required to meet the case definition of AFFs. ${ }^{15}$

Cortical thickness - an increase in cortical thickness of the diaphysis of the femur(s). Symptoms- the presence of unilateral or bilateral prodromal symptoms (e.g. dull or aching pain in the thigh or groin). Bilateral occurrencebilateral incomplete or complete femoral diaphysis fractures and delayed fracture healing.

The first report of the ASBMR task force (2010 case definition) previously made reference to some named classes of drugs that were recognised to be associated with potentially increased risk of femoral fractures (e.g. drugs like bisphosphonates, glucocorticoids, and proton pump inhibitors - PPIs). ${ }^{14}$ In addition, the task force's first report cited some comorbid medical conditions that are also associated with increased fracture risk e.g. diabetes mellitus, rheumatoid arthritis, vitamin D deficiency, and hypophosphatasia). ${ }^{14}$

In the updated or second report of the ASBMR task force, the authors removed both the citation of some specific comorbid conditions and specified medication exposures to the risks of AFFs. ${ }^{15}$ The second report clarified the rationale for these actions. The task force indicated that they had considered these points, and made a determination that is was more appropriate for studies to seek these associations to AFFs, rather than for the case definition of AFFs to be directly linked to these conditions and medications. ${ }^{15}$

Also, in the updated ASBMR task force's second report, further stated exclusions from the case definitions of AFFs were: femoral neck fractures, intertrochanteric fractures with spiral subtrochanteric extension, pathological fractures occurring in association with primary bone malignancy or metastatic bone tumours, and also periprosthetic fractures. ${ }^{15}$ It is important to highlight that although the ASBMR second task force's case definition of AFFs specifically excluded periprosthetic 
fractures, some other authors have cited emerging data suggesting that AFF-type events may occur in this context as well. ${ }^{5}$

\section{The index cases}

The index case report had identified risk factors for osteoporosis which included her post-menopausal state (aged 72 years), use of omeprazole $20 \mathrm{mg}$ once daily, and previous long-term steroid (prednisolone) therapy for PMR. She had no other noted risk factors for pathological fractures e.g. cigarette smoking or history of malignancy. In the absence of previous femoral operations, these new fractures were also not periprosthetic in nature.

She had been taking 70mg once weekly (generic) oral alendronate on a regular basis for the ten years preceding her presentation with groin and thigh pains. The pain was characterised as being aching in nature. The pains had arisen in the absence of falls or trauma. Plain radiology noted features consistent with impending AFF in the subtrochanteric regions.

The index case meets the ASBMR definition of having the 'required feature' of fractures occurring along the femoral shaft, and specifically occurring in the subtrochanteric regions.

This reported case also exceeds the minimum 4 out of 5 'major features' of the ASBMR (no trauma; fracture lines originating in the lateral cortices, incomplete fractures involve the lateral cortices only, non-comminuted nature, presence of localised periosteal thickening of the lateral cortices at the sites of the fractures- i.e. radiologic appearance of 'beaking' or 'flaring').

Furthermore, this case also meets some of the ASBMR case definition's 'minor features' (e.g. the presence of bilateral prodromal features of pain in the thighs; radiologic signs of incomplete or impending AFFs occurring bilaterally).

Although the patient in this report had evolving prodromal features of bilateral dull pain in the left groin, and later involving both thighs, it is important for clinicians to recognise that patients on bisphosphonates may sustain AFFs in the absence of such symptoms. It is also important to recognise that where present, prodromal symptoms are actually currently classified as minor features (for the purposes of the ASBMR's case definition of AFFs).

In scenarios where a patient on bisphosphonate therapy is noted to have AFF compatible features in one limb (e.g. involving the subtrochanteric region or the femoral shaft), it would be sensible and advisable that the contralateral femur is also subject to radiologic imaging. The latter allows for early assessment for any radiological evidence of contralateral cortical thickening, stress reactions to localised areas of bone, or incomplete fractures (which may exist even in the absence of symptoms).

The patient was admitted directly under the care of specialist consultant orthopaedic surgeons. Liaison input was provided by a consultant physician with an interest in orthogeriatric medicine and who subsequently managed her period of inpatient orthogeriatic rehabilitation. In this patient, the alendronic acid was discontinued. Given the context, a clinical decision was taken that it would not be appropriate to consider a 'medication re-challenge' by reintroduction of the alendronate after a period of discontinuation, nor was it appropriate to resume this specifFic treatment after a 'drug holiday.'

At the request of the managing orthogeriatric medical consultant, a clinical pharmacist assisted with the completion of an online formal ADR notification record (citing the prolonged use of oral alendronic acid in association with a presentation with bilateral atraumatic AFFs). This was completed via a nationally applicable system i.e. yellow card scheme (United Kingdom). ${ }^{17}$ This patient was not noted to have other significant ADRs linked to bisphosphonate use e.g. osteonecrosis of the jaw (dental osteonecrosis), or osteonecrosis of the external auditory canal. ${ }^{1,13}$

The patient successfully underwent two planned and sequential orthopaedic surgeries, within a pre-scheduled one-week interval. These involved surgical insertion of bilateral intertrochanteric antegrade nails (inter-TAN) to the femoral bones. ${ }^{18}$ Thereafter, she progressed well with a short period of inpatient rehabilitation, and was discharged home. A referral was made for an outpatient DEXA scan.19 The aim of the DEXA scan was to support the re-evaluation of her BMD. If appropriate, she could then be considered for a substitution from the previous bisphosphonate therapy to the option of subcutaneous denosumab (the latter to be considered as a possible alternative treatment for her osteoporosis). ${ }^{19}$

\section{Applying ADR causality assessment systems to the index case}

The routine use of validated causality assessment systems (CAS) may improve the objectivity and transparency during the reporting of suspected ADR-related cases, and as part of pharmacovigilance case assessments. ${ }^{20-22}$ For example, applying the Naranjo adverse drug reaction probability Scale to this case translates into a score of $6 .^{20}$ This equates to a 'probable' ADR classification. ${ }^{20}$ Using an alternative validated CAS, i.e. the WHO-UMC method also derives a 'probable/likely' ADR classification for this index report. ${ }^{23}$

\section{CONCLUSION}

This report describes the case of an older female patient with clinical symptoms and radiological evidence suggestive of bilateral AFFs arising in association with 
the prolonged use (ten years) of oral alendronate sodium. The case illustrates the application of the ASBMR in the case definition of this report. The report is a clinical reminder of the need to undertake staged reviews of the use of bisphosphonates such as alendronic acid to monitor for the development of any possible ADRs. This is especially important where early symptom profiles (e.g. dull or aching pains in the groin, hip or thigh) may highlight the presence of imminent 'higher risk' ADRs, or might flag up the possible development of major complications of prolonged treatment with bisphosphonates, such as AFFs. Furthermore, the report offers a helpful clinical lesson for a range of clinicians e.g. community-based/primary care physicians, or hospital-based doctors (including orthopaedic surgeons, geriatricians, rheumatologists, general physicians, radiologists, etc). The report is also relevant to other clinicians who may practice within approved or extended roles e.g. prescribers from backgrounds in clinical pharmacy, nurse specialists (e.g. osteoporosis), nurse practitioners or advanced nurse practitioners, physician associates, etc. In addition to the encouraged use of a CAS, clinicians and prescribers should familiarise themselves with relevant ADR reporting systems for their respective countries of practice. Finally, prescribers should routinely engage in the reporting of ADRs as this can augment pharmacovigilance.

\section{Funding: No funding sources}

Conflict of interest: None declared

Ethical approval: Not required

\section{REFERENCES}

1. British National Formulary. National Institute for Health \& Care Excellence. (C) NICE 2019. Available at: https://bnf.nice.org.uk/drug/alendronic-acid.html Accessed on 02 September 2020.

2. National institute for health and care excellence. Bisphosphonates for treating osteoporosis (TA464). 2017, last updated 2019. Available at https://www.nice.org.uk/guidance/ta464. Accessed on 02 September 2020.

3. Wells G, Cranney A, Peterson J, Boucher M, Shea B, Robinson $\mathrm{V}$, et al. Alendronate for the primary and secondary prevention of osteoporotic fractures in postmenopausal women. Cochrane Database Syst Rev 2008;(1):CD001155.

4. Black DM, Geiger EJ, Eastell R, Vittinghoff E, Li BH, Ryan DS, et al. Atypical Femur Fracture Risk versus Fragility Fracture Prevention with Bisphosphonates. N Engl J of Med. 2020;383(8):74353.

5. Starr J, Tay YK, Shane E. Current understanding of epidemiology, pathophysiology, and management of atypical femur fractures. Curre Osteoporo Repor. 2018;16(4):519-29.

6. Carrarino JE. Bisphosphonates and atypical femoral fractures. J Nurs Practition. 2015;11(4):389-96
7. Odvina CV, Levy S, Rao S, Zerwekh JE, Rao DS. Unusual mid-shaft fractures during long-term bisphosphonate therapy. Clinic Endocrinol. 2010;72(2):161-8.

8. Temponi EF, Carvalho Junior LH, Costa LP. Atypical femoral fracture due to chronic use of bisphosphonates: case report. Brazi Orthoped Magaz. 2015;50(4):482-5.

9. Reddy SV. Long term bisphosphonate therapy \& atypical femoral fractures: a word of caution. Indian J Med Res. 2014;140(1):8-10.

10. Thompson RN, Phillips JR, McCauley SH, Elliott JR, Moran CG. Atypical femoral fractures and bisphosphonate treatment: experience in two large United Kingdom teaching hospitals. J Bone Joint Surg Br. 2012;94(3):385-90.

11. Jain TP, Thorn M. Atypical femoral fractures related to bisphosphonate therapy. Indian J Radiol Imaging. 2012;22(3):178-181. doi:10.4103/0971-3026.107178

12. Schilcher J, Michaëlsson K, Aspenberg $P$. Bisphosphonate use and atypical fractures of the femoral shaft. N Engl J of Med. 2011 May 5;364(18):1728-37.

13. Ogundipe OA. A case of variegate porphyria in association with coeliac disease and bisphosphonate associated dental osteonecrosis. J Clin Med Res. 2009 Dec;1(5):292.

14. Shane E, Burr D, Ebeling PR, Abrahamsen B, Adler RA, Brown TD, et al. Atypical subtrochanteric and diaphyseal femoral fractures: report of a task force of the American Society for Bone and Mineral Research. J Bone Miner Res. 2010;25(11):2267-94.

15. Shane E, Burr D, Abrahamsen B, Adler RA, Brown TD, Cheung AM, et al. Atypical subtrochanteric and diaphyseal femoral fractures: second report of a task force of the American Society for Bone and Mineral Research. J Bone Miner Res. 2014;29(1):1-23.

16. LeBlanc ES, Rosales AG, Black DM, Genant HK, Dell RM, Friess DM, et al. Evaluating atypical features of femur fractures: how change in radiological criteria influenced incidence and demography of atypical femur fractures in a community setting. J Bon Miner Resear. 2017;32(11):2304-14.

17. Yellow Card. (C) Crown Copyright 2020. Available at https://yellowcard.mhra.gov.uk/. Accessed on 02 September 2020.

18. National institute for health and care excellence. Hip fracture: Management (CG124). 2011, last updated $2017 . \quad$ https://www.nice.org.uk/guidance/cg124. Accessed on 2 Sept. 2020.

19. Scottish Intercollegiate Guidelines Network. Management of osteoporosis and the prevention of fragility fractures (SIGN 142). 2015, last updated June 2020. Available at https://www.sign.ac.uk/ Accessed on 02 September 2020.

20. Naranjo CA, Busto U, Sellers EM, Sandor P, Ruiz I, Roberts EA, et al. A method for estimating the probability of adverse drug reactions. Clin Pharmacol Ther. 1981;30 (2): 239-45. 
21. Busto U, Naranjo CA, Sellers EM. Comparison of two recently published algorithms to assess the probability of adverse drug reactions. $\mathrm{Br} \mathrm{J}$ Clin Pharmacol. 1982;13(2): 223-7.

22. Kramer MS, Leventhal JM, Hutchinson TA, Feinstein AR: An algorithm for the operational assessment of adverse drug reactions. I. Background, description, and instructions for use. J Americ Medic Assoc. 1979;242(7):623-32.

23. The use of the WHO-UMC system for standardised case causality assessment. Uppsala Monitoring
Centre. Available at https://www.who.int /medicines/areas/quality_safety/safety_efficacy/WH Ocausality_assessment.pdf. Accessed on 02 September 2020 .

Cite this article as: Ogundipe OA. Nontraumatic bilateral atypical femoral fractures associated with long-term alendronate therapy. Int $\mathrm{J}$ Basic Clin Pharmacol 2020;9:1737-43. 